\title{
Genome Sequence Resource of 'Candidatus Liberibacter asiaticus' from Diaphorina citri Kuwayama (Hemiptera: Liviidae) in Colombia
}

\author{
Yanjing Wang, ${ }^{1}$ Takumasa Kondo, ${ }^{2}$ Yurong He, ${ }^{3}$ Zhuohuai Zhou, ${ }^{1}$ and Jinming Lu ${ }^{4, \dagger}$ \\ ${ }^{1}$ College of Agriculture and Food Sciences, Zhejiang Agriculture \& Forestry University, Lin'an, Zhejiang \\ 200300, China \\ ${ }^{2}$ Corporación Colombiana de Investigación Agropecuaria (AGROSAVIA), Laboratorio de Entomología, \\ Centro de Investigación Palmira, Colombia \\ ${ }^{3}$ Department of Entomology, South China Agricultural University, Guangzhou, Guangdong 510642, \\ China \\ ${ }^{4}$ College of Forestry and Biotechnology, Zhejiang Agriculture \& Forestry University, Lin'an, Zhejiang \\ 200300, China
}

\begin{abstract}
'Candidatus Liberibacter asiaticus' (CLas) is an unculturable phloem-restricted $\alpha$ proteobacterium associated with huanglongbing (HLB). Here, we provide the genome sequence of CLas strain CoFLP1 from its insect vector Diaphorina citri (Hemiptera: Liviidae) collected in the department of La Guajira, Colombia. The CoFLP1 strain is composed of $1,231,639$ bp with $\mathrm{G}+\mathrm{C} 36.5 \%$ content. This study reports the first $C$ Las genome sequence from Colombia, which will add to CLas genome resources and help to elucidate our understanding of the introduction pathway of HLB in South America.
\end{abstract}

Citrus huanglongbing (HLB), also known as citrus greening disease, is a highly destructive disease worldwide associated with 'Candidatus Liberibacter asiaticus' (CLas), 'Ca. L. americanus' (CLam), and 'Ca. L. africanus' (CLaf) (Bové 2006; Lin 1956). The Asian citrus psyllid, Diaphorina citri Kuwayama (Hemiptera: Liviidae), is the main vector of $C$ Las, the most widely distributed variant. In Colombia, South America, D. citri was first reported by the Colombian Agricultural Institute in the departments of Valle del Cauca and Tolima in 2007 (Ebratt-Ravelo et al. 2011). The insect pest then rapidly spread to all of the Andean and Orinoquia regions of Colombia (Ebratt-Ravelo et al. 2011; King 2012). Currently, D. citri is widely distributed in 26 departments of Colombia, i.e., Antioquia, Arauca, Atlántico, Bolívar, Boyacá, Caldas, Caquetá, Casanare, Cauca, Cesar, Córdoba, Cundinamarca, Huila, La Guajira, Magdalena, Meta, Nariño, Norte de Santander, Quindío, Risaralda, San Andrés, Santander, Sucre, Tolima, Valle del Cauca, and Vichada (Kondo et al. 2017).

In 2015, HLB was first detected in the northern regions of Colombia, in the department of La Guajira, by the Colombian Agricultural Institute (ICA 2015), which raised an alarm to citrus production in the national territory and neighboring countries. Currently, HLB has spread to six northern departments of Colombia, i.e., Atlántico, Bolívar, César, La Guajira, Magdalena, and Norte de Santander (ICA 2018). Little is known about the origin and diffusion of this disease in Colombia. The difficulty of isolation and pure culture limits research on the biology of HLB (Duan et al. 2009). Along with the advance in sequencing technology, more information could be obtained from the genome of CLas (Liu et al. 2020; Thapa et al. 2020). There are currently 26 whole-genome sequences of $C$ Las strains available in GenBank, limited to China, Japan, Mexico, and the U.S.A. In this study, we performed whole-genome sequencing of a CLas strain from Colombia.

${ }^{\dagger}$ Corresponding author: J. M. Lu; $67698321 @$ qq.com

The author(s) declare no conflict of interest.

Accepted for publication 28 July 2020.
Funding

This study was supported by Guangdong Provincial Special Fund for Modern Agriculture Industry Technology Innovation Teams (2019KJ125).

\section{Keywords}

'Candidatus Liberibacter asiaticus', citrus huanglongbing, genome, Colombia 
Table 1. Mapping information of 'Candidatus Liberibacter asiaticus' strain CoFLP1

\begin{tabular}{lccc} 
Reference sequence & Consensus (bp) & Total read count & Average nucleotide coverage \\
A4 $(1,233,514$ bp) & $1,209,458$ & $2,274,445$ & 257.85 \\
SC1 (40,048 bp) & 35,627 & 29,118 & 103.38 \\
SC2 (38,997 bp) & 19,607 & 7,289 & 25.23 \\
P-JXGC-3 (31,449 bp) & 17,177 & 6,904 & 30.84 \\
\hline
\end{tabular}

D. citri was collected from shoots of Citrus latifolia Tanaka (Rutaceae) from trees with clear symptoms of HLB (i.e., asymmetrical pattern of blotchy yellowing or mottling of the leaves) in Municipio Dibulla, Vereda Palomino, La Guajira, Colombia $\left(11^{\circ} 16^{\prime} \mathrm{N} 73^{\circ} 18^{\prime} \mathrm{W}\right.$, ca. $2 \mathrm{~m}$ asl) by T. Kondo in October 2017. Total DNA was extracted from a single adult using the Qiagen DNeasy Blood \& Tissue Kit (Qiagen, Valencia, CA, U.S.A.). CLas presence was confirmed by a real-time PCR method targeting the 16S rRNA gene (Li et al. 2006). A library was prepared using the Illumina TruSeq V2 paired-end library preparation kit (300-bp insert size) (Illumina, San Diego, CA). Genome sequencing was performed on HiSeq PE150 Illumina platform (Illumina Inc., San Diego, CA).

A total of $47,126,744$ reads with an average size of $150 \mathrm{bp}$ per read were generated. The reads were trimmed to remove adapters and bases below a quality score of 30 using Trimmomatic 0.36 (Bolger et al. 2014). The reads were mapped against genome sequence of CLas strain A4 (GenBank: CP010804), SC1 (GenBank: HQ377372), SC2 (GenBank: HQ377373), and P-JXGC-3 (GenBank: KY661963) using CLC Genomics Workbench 12 (Qiagen). Then de novo assembly was performed using SPAdes 3.14.1 (Bankevich et al. 2012). A total of 22 contigs (over $1,000 \mathrm{bp}$ ), ranging from 1,159 to $367,773 \mathrm{bp}$, were obtained through BLAST+2.9.0 (Camacho et al. 2009) against the local database using CLas A4 strain (GenBank: CP010804). Gap closure was conducted by PCR with specific primer sets designed based on the adjacent sides of two contigs and Sanger sequencing. The draft genome of CoFLP1 is $1,231,639$ bp in length, with the GC content of $36.5 \%$. The genome was annotated using the RAST server (https://rast.nmpdr.org) (Aziz et al. 2008), with 1,190 open reading frames (ORFs) and 53 RNA genes. The CoFLP1 strain was confirmed as having Type 1-like (SC1) prophages (Table 1).

This study reports the first whole genome sequence of a CLas strain from South America, which will provide a reference for HLB research in Colombia. The CoFLP1 strain will contribute to studies on CLas genetic diversity worldwide and precision control strategies of HLB. It will also help to elucidate our understanding of the introduction pathway of HLB in South America.

Accession Number. This whole-genome shotgun project has been deposited at DDBJ/ ENA/GenBank under the accession number CP054558.

\section{Literature Cited}

Aziz, R. K., Bartels, D., Best, A. A., De Jongh, M., Disz, T., Edwards, R. A., Formsma, K., Gerdes, S., Glass, E. M., Kubal, M., Meyer, F., Olsen, G. J., Olson, R., Osterman, A. L., Overbeek, R. A., McNeil, L. K., Paarmann, D., Paczian, T., Parrello, B., Pusch, G. D., Reich, C., Stevens, R., Vassieva, O., Vonstein, V., Wilke, A., and Zagnitko, O. 2008. The RAST server: rapid annotations using subsystems technology. BMC Genomics 9:75.

Bankevich, A., Nurk, S., Antipov, D., Gurevich, A. A., Dvorkin, M., Kulikov, A. S., Lesin, V. M., Nikolenko, S. I., Pham, S., and Prjibelski, A. D. 2012. SPAdes: a new genome assembly algorithm and its applications to single-cell sequencing. J. Comput. Biol. 19:455-477.

Bolger, A. M., Lohse, M., and Usadel, B. 2014. Trimmomatic: a flexible trimmer for Illumina sequence data. Bioinformatics 30:2114-2120.

Bové, J. M. 2006. Huanglongbing: A destructive, newly emerging, century-old disease of citrus. J. Plant Pathol. 88:7-37.

Camacho, C., Coulouris, G., Avagyan, V., Ma, N., Papadopoulos, J., Bealer, K., and Madden, T. L. 2009. BLAST+: architecture and applications. BMC Bioinformatics 10:421.
Duan, Y. P., Zhou, L. J., Hall, D. G., Li, W. B., Doddapaneni, H., Lin, H., Liu, L., Vahling, C. M., Gabriel, D. W., Williams, K. P., Dickerman, A., Sun, Y. J., and Gottwald, T. 2009. Complete genome sequence of citrus huanglongbing bacterium, 'Candidatus Liberibacter asiaticus' obtained through metagenomics. Mol. Plant-Microbe Interact. 22:1011-1020.

Ebratt-Ravelo, E. E., Rubio-Gónzález, L. T., Costa, V. A., Castro-Ávila, A. P., Zambrano-Gómez, E. M., and Ángel-Díaz, J. E. 2011. Diaphorina citri (Kuwayama, 1907) and Tamarixia radiata (Waterson, 1922) in citrus crops of Cundinamarca, Colombia. Agron. Colomb. 29:487-493.

Instituto Colombiano Agropecuario (ICA). 2015. Resolución No. 00002390. Instituto Colombiano Agropecuario, Bogotá, Colombia. https://diario-oficial.vlex.com.co/ $\mathrm{vid} /$ resolucion-numero-00002390-2015-589641750

Instituto Colombiano Agropecuario (ICA). 2018. Resolución 6415 de 2018. Diario Oficial No. 50.618 de 8 de junio de 2018. Instituto Colombiano Agropecuario, Bogotá, Colombia. Available at: https://icbf.gov.co/cargues/avance/docs/resolucion_ica_26415_2018.htm.

King, W. 2012. Dispersión de Diaphorina citri (Hemiptera: Psyllidae) en el departamento del Tolima (Colombia). Rev. Tumbaga. 7:51-60. 
Kondo, T., García-Córdoba, C. Y., Sotelo-Cardona, P. A., and Ramos-Villafañe, Y. P. 2017. Capítulo VI. Diaphorina citri Kuwayama, hospedante de Tamarixia radiata (Waterston). Pages 67-78 in: Protocolo de cría y liberación de Tamarixia radiata Waterston (Hymenoptera: Eulophidae). T. Kondo, ed. Corporación Colombiana de Investigación Agropecuaria (Corpoica), Mosquera, Colombia.

Li, W. B., Hartung, J. S., and Levy, L. 2006. Quantitative real-time PCR for detection and identification of Candidatus Liberibacter species associated with citrus huanglongbing. J. Microbiol. Methods 66:104-115.
Lin, K. H. 1956. Observation on yellow shoot of citrus. Acta Phytopathol. Sin. 2:1-11.

Liu, K., Atta, S., Cui, X., Zeng, C., Chen, J. C., Zhou, C. Y., and Wang, X. 2020.

Genome sequence resources of two 'Candidatus Liberibacter asiaticus' strains from Pakistan. Plant Dis. 104:2048-2050.

Thapa, S. P., De Francesco, A., Trinh, J., Gurung, F. B., Pang, Z., Vidalakis, G., Wang, N., Ancona, V., Ma, W., and Coaker, G. 2020. Genome-wide analyses of Liberibacter species provides insights into evolution, phylogenetic relationships, and virulence factors. Mol. Plant Pathol. 21: 716-731. 